\title{
The ASTRI-Horn Dual-Mirror Small-Size Cherenkov Telescope: recent updates, first results and outlook
}

\author{
Cornelia Arcaro* \\ Centre for Space Research, North-West University, Potchefstroom 2520, South Africa and INAF \\ Osservatorio Astronomico di Padova, vicolo dell'Osservatorio 5, 35122, Padova, Italy \\ E-mail: cornelia.arcaro@nwu.ac.za
}

for the CTA ASTRI project

The ASTRI project represents an effort of an international collaboration led by the Istituto Nazionale di Astrofisica, Italy, in conjunction with the Universidade de Sao Paulo, Brazil, and the North-West University, South Africa, aiming at assessing the technology of an array of smallsize Cherenkov telescopes (SSTs). In this respect, the ASTRI-Horn telescope, an end-to-end prototype, has been installed in Sicily, on the slopes of the Etna volcano at $1735 \mathrm{~m}$ a.s.l. The telescope is characterized by a dual-mirror Schwarzschild-Couder design that covers a wide field of view and favors gamma-ray observations in the range from few TeVs up to tens of TeV. Its curved focal-plane camera consists of Silicon-photomultipliers, which are controlled by fast front-end electronics. The complete end-to-end approach includes the implementation of calibration as well as control and acquisition systems, together with the data reduction, archiving and analysis software. The ASTRI collaboration has successfully completed the commissioning and science verification phase of the prototype, including observation of the Crab Nebula. A mini-Array composed of nine ASTRI telescopes is being developed and operated by INAF in the context of the preparatory effort for the proposed participation in the Cherenkov Telescope Array (CTA). Apart from a few minor changes implemented to improve the telescope design, the mini-array telescopes will be very similar to the ASTRI-Horn telescope. In this contribution an overview of the main features and performance of the ASTRI-Horn telescope is presented together with recent updates and first scientific results. In addition to this, the technological evolution towards the ASTRI mini-array and its scientific expectations are outlined.

High Energy Astrophysics in Southern Africa - HEASA2019

28-30 August, 2019

Swakopmund, Namibia

* Speaker. 


\section{Introduction}

The most sensitive instruments to very-high-energy (VHE, $E>50 \mathrm{GeV}$ ) gamma rays are ground-based imaging atmospheric Cherenkov telescopes (IACTs; e.g., see [1] for a review) and are most effectively operated when several of these are spaced several tens of meters apart and pointed to the same sky position. In this way, they observe the same event with different orientations allowing its stereoscopic imaging and reconstruction.

ASTRI-Horn [2] is the end-to-end prototype IACT developed by the Italian National Institute of Astrophysics (INAF) as a proposed component of the Cherenkov Telescope Array Observatory $\left(\mathrm{CTAO}^{1}\right)$. The prototype has been installed at Serra La Nave, Mount Etna, Sicily, and its scientific verification phase was concluded in 2019. The telescope is based on a Schwarzschild-Couder (SC) design proposed by Vassiliev et al. [3] that is characterized by a dual-mirror optical system and a curved photon detector placed in the focal plane. This layout allows the contemporaneous correction for spherical, coma and astigmatism aberrations on a large field of view. Given its design, the ASTRI-Horn telescope is optimized for the detection of the high end of the VHE energy range (5 $\mathrm{TeV}<E<300 \mathrm{TeV}$; [4]).

\section{The ASTRI-Horn Telescope}

The ASTRI-Horn telescope is a compact system covering a total effective field of view (FoV) of $7.6^{\circ}$. Its design is characterized by a $4 \mathrm{~m}$-diameter primary (M1) and a $1.8 \mathrm{~m}$-diameter secondary mirror (M2), with a primary-to-secondary distance of $3 \mathrm{~m}$, and a secondary-to-camera distance of $0.52 \mathrm{~m}[5,6]$. It adopts an altitude-azimuthal design, with the mirror dish mounted on the azimuth fork.

The ASTRI-Horn features a novel camera design that exploits the characteristics of the telescope's SC layout, leading to a very compact fine-pixelized camera $(0.52 \mathrm{~m} \times 0.66 \mathrm{~m} \times 0.56 \mathrm{~m}$; [7]). Based on a custom peak-detector operation mode, the read-out electronics represent a solution that relies on a CITIROC ASIC (Application Specific Integrated Circuit; [8]). A particular operating mode of the read-out electronics is the so-called 'variance' technique [7, 8, 9], for which the electric signal generated by each pixel that is not triggered by the first level trigger is continuously sampled. While the sequence of ADC (Analog to Digital Converter) values obtained is constant with time, its variance is proportional to the photon flux impinging on the pixel. The acquisition of the variance data is performed in parallel to the normal data acquisition. The method has several applications ranging from validating the pointing accuracy of the telescope and determining the night sky background to monitoring the mirror alignment.

\section{Comissioning and validation of the ASTRI-Horn telescope}

Since its inauguration in 2014, an extensive set of tests was performed, to characterize and subsequently monitor the performance of the ASTRI-Horn telescope [10].

The validation of the optical design has been conducted during a dedicated campaign in Autumn 2016. The optical point spread function (PSF) has been determined as a function of the

\footnotetext{
${ }^{1}$ https://www.cta-observatory.org/
} 
position along the telescope's field of view and its elevation [11, 12], showing that the required specification of a flat PSF of $\sim 10$ arcminutes along a large field of view is fulfilled. The monitoring of the mirror reflectivity is crucial for evaluating the degradation of their reflective coating due to the environmental impact. For this reason, the reflectivity of the M1 and M2 mirrors has been measured at different times with a spectrometer, indicating that the ageing of the coating was faster than expected, especially for the secondary mirror, due to the aggressive volcanic atmosphere with frequent emission of sulphur compounds [13].

A second method to monitor the mirror degradation and, more generally, for the calibration of the telescope's optical throughput with high precision, based on the analysis of muon-induced Cherenkov events, was also investigated [14]. The data used were taken between December 2018 and March 2019 and their analysis confirmed the mirror reflectivity degradation during observations in March 2019 after a strong Etna eruption.

In order to evaluate the telescope's tracking and pointing precision, dedicated observations have been carried out between November 2017 and January 2018. Both pointing and tracking precision were found to fulfill the needed requirements, with a root mean square of $\sim 5$ to 6 arcseconds.

The integration of the ASTRI camera at the telescope was carried out in May 2017. Since then, the camera underwent engineering tests, and some scientific runs were performed. First trigger rate scans have been recorded during moonless nights while pointing the telescope at different sky positions. The analysis of these scans indicated the camera's working point for the detection of Cherenkov light to be at $\sim 11$ photoelectrons (phe), meaning that the trigger rate in the region dominated by air-shower events was lower than expected from previous measurements in the laboratory, due to a reduced telescope efficiency at that time that was associated with the mirror degradation. On the other hand, the trigger threshold was found to be higher due to the local night sky background being higher than expected $[7,15]$.

The variance mode of the camera [7, 8, 9] has been evaluated in 2018 and showed its potential in assessing the telescope's pointing and checking the optical alignment of the M1 segments [11]. In addition, the variance data have been proven to identify potential pointing errors such as an offset with respect to the camera center with an accuracy of up to few arcseconds, as well as to be a powerful tool to monitor the telescope's PSF with high spatial resolution and a large dynamical range.

In December 2018, 12.4 hours of gamma-ray data on the Crab Nebula and 12.0 hours of dedicated off-target data were collected, respectively, resulting in a detection of this well-known VHE emitter above $\sim 3 \mathrm{TeV}$ [15], despite the reduced efficiency of the telescope as well as a non-optimal tuning of the camera.

\section{Science with the ASTRI mini-array}

Besides the purpose to verify technological and operational aspects, the ASTRI mini-array is designed to perform astrophysical observations [16]. Based on Monte Carlo studies performed with the A-SciSoft package [17], the ASTRI mini-array could push the next generation of IACTs towards being sensitive to gamma rays above $10 \mathrm{TeV}$ up to about $100 \mathrm{TeV}$. Several studies have been conducted to evaluate its potential science cases, by simulating the $\mathrm{TeV}$ gamma-ray emission 
of promising sources accessible to the ASTRI mini-array from the Southern Hemisphere, considering the Atacana Desert in Chile as potential site. Recently, the Canarian Island Tenerife has been evaluated as an alternative site from which the northern sky will be accessible and new studies on promising objects are currently being conducted. The studies presented in the following have still been made under the assumption of a southern site.

The ASTRI mini-array will exploit its sensitivity and extended spectral range to investigate the emission of prominent sources with hard spectra such as galactic sources like young ( $\sim 2000$ years) pulsar wind nebulae (e.g., Crab Nebula and Vela-X) and supernovae remnants (SNRs; e.g., Velajunior, RX J1713.7-3946, MSH 15-52; [18]). Being optimised for the highest energies and featuring a large FoV in combination with a good angular resolution, the ASTRI mini-array may contribute to the understanding of the origin of cosmic rays (CR) by distinguishing between hadronic and leptonic emission scenarios for these kinds of sources. More importantly, by observations of the high-energy tail of the spectra of VHE emitting sources located at the Galactic Centre, the mini-array may investigate the existence of cosmic rays with energies of the order of $\sim 10^{15} \mathrm{eV}$. This could confirm Sagittarius A* as a PeVatron, as suggested by H.E.S.S. [19].

Other promising targets for the ASTRI mini-array are extragalactic sources with hard spectra, for example extreme blazars like 1ES 229+200. Studies may shed light on the mechanism of gamma-ray emission, which may eventually involve ultra-high-energy cosmic rays (UHECRs; [20]). Cosmological studies closely related with this type of sources include searches for anomalies in the opacity of the Universe to VHE gamma rays due to the extragalactic background light (EBL) by the existence of axion-like particles (ALPs) and attempts at constraining the intergalactic magnetic field (IGMF). In addition to this, EBL studies at wavelengths $>10 \mu \mathrm{m}$ can be carried out by observing nearby so-called high-frequency-peaked BL Lac objects (e.g., Mrk 421 and Mrk 501) and radio galaxies (e.g., M 87), where the EBL absorption is only significant above $\sim 30 \mathrm{TeV}$ [21].

\section{Conclusion}

The entire ASTRI-Horn system has been developed following an end-to-end approach. During an extensive validation phase, which concluded with the detection of the Crab Nebula, it has been demonstrated to fulfill expectations and requirements, while the next phase towards the implementation of a mini-array of nine ASTRI telescopes has already been entered. The ASTRI mini-array will allow early science operations extending the observation of gamma rays in an energy range previously largely unexplored by current IACT facilities.

\section{Acknowledgement}

This work is supported by the Italian Ministry of Education, University, and Research (MIUR) with funds specifically assigned to the Italian National Institute of Astrophysics (INAF) for the Cherenkov Telescope Array (CTA), and by the Italian Ministry of Economic Development (MISE) within the 'Astronomia Industriale' program. We acknowledge support from the Brazilian Funding Agency FAPESP (Grant 2013/10559-5) and from the South African Department of Science and Technology through Funding Agreement 0227/2014 for the South African Gamma-Ray Astronomy 
Programme. We gratefully acknowledge support from the agencies and organizations listed under Funding Agencies at this website: http://www.cta-observatory.org.

\section{References}

[1] Rieger, F.M., de Ona-Wilhelmi, E., and Aharonian, F. A., TeV Astronomy, Frontiers of Physics, Volume 8 Issue 6 (2013), 714 [DOI:10.1007/s11467-013-0344-6]

[2] Maccarone, M.C., et al., ASTRI for the Cherenkov Telescope Array, in Proceedings of 35th International Cosmic Ray Conference, (2017), [doi:10.22323/1.301.0855]

[3] Vassiliev, V., Fegan, S., and Brousseau, P., Wide field aplanatic two-mirror telescopes for ground-based g-ray astronomy, Astroparticle Physics, 28 (2007), 10 [DOI:10.1016/j.astropartphys.2007.04.002]

[4] Pareschi G., et al., The ASTRI SST-2M prototype and mini-array for the Cherenkov Telescope Array (CTA), in proceedings of SPIE conferences, 9906 (2016), ID 99065T [DOI:10.1117/12.2232275]

[5] Canestrari, R., et al., The ASTRI SST-2M Prototype: Structure and Mirror, in proceedings of the 33rd ICRC, (2013), [arXiv: 1307.4851]

[6] Rodeghiero, G., et al., Qualification and testing of a large hot slumped secondary mirror for Schwarzschild-Couder imaging air Cherenkov telescopes, Publications of the Astronomical Society of the Pacific, 128 (2016), 963 [DOI:10.1088/1538-3873/128/963/055001]

[7] Catalano, O., et al., The ASTRI camera for the Cherenkov Telescope Array, in proceedings of SPIE conferences, 10702 (2018), ID 1070237 [DOI:10.1117/12.2314984]

[8] Sottile, G., et al., ASTRI SST-2M Camera Electronics, in proceedings of SPIE conferences, 9906 (2016), ID 99063D [DOI:10.1117/12.2232464]

[9] Segreto, A., et al., Calibration and monitoring of the ASTRI-Horn telescope by using the night-sky background measured by the photon-statistics ( "variance") method, in proceedings of the 37th ICRC (2019), 791 [arXiv:1909.08750]

[10] Canestrari, R., et al., ASTRI SST-2M prototype for the Cherenkov Telescope Array: status after the commissioning phase of the telescope, in proceedings of SPIE conferences, 10399 (2017), ID 1039904 [DOI:10.1117/12.2276767]

[11] Giro E., et al., First optical validation of a Schwarzschild Couder telescope: the ASTRI SST-2M Cherenkov telescope, Astronomy \& Astrophysics, 608 (2017), A86 [DOI:10.1051/0004-6361/201731602]

[12] Canestrari, R., et al., The ASTRI SST-2M prototype for the Cherenkov Telescope Array: opto-mechanical performance, in proceedings of SPIE conferences, 9906 (2017), ID 990619 [DOI:10.1117/12.2232270]

[13] Scuderi, S., et al., From the Etna volcano to the Chilean Andes: ASTRI end-to-end telescopes for the Cherenkov Telescope Array, proceedings of SPIE conferences, 10700 (2018), ID $107005 \mathrm{Z}$ [DOI:10.1117/12.2312453]

[14] Mineo, T., et al., Using muon rings for the optical calibration of the ASTRI telescopes for the Cherenkov Telescope Array in Proceedings of the SPIE conference, 9913 (2016), ID 99132R [DOI:10.1117/12.2232256] 
[15] Lombardi, S., et al First detection of the Crab Nebula at TeV energies with a Cherenkov telescope in dual-mirror Schwarzschild-Couder configuration: the ASTRI-Horn telescope, (2019), [DOI:10.1051/0004-6361/201936791]

[16] Pareschi G., Bonnoli, G., and Vercellone, S., The mini-array of ASTRI SST-2M telescopes, precursors for the Cherenkov Telescope Array, Journal of Physics: Conference Series, 718 (2016), ID 052028 [DOI:10.1088/1742-6596/718/5/052028]

[17] Lombardi, S., et al., ASTRI data reduction software in the framework of the Cherenkov Telescope Array, in proceedings of SPIE conferences, 10707 (2018), ID 107070R [DOI:10.1117/12.2311293]

[18] Burtovoi, A., et al., Prospects for PWNe and SNRs science with the ASTRI mini-array of pre-production small-sized telescopes of the Cherenkov telescope array, in AIP Conference Proceedings, 1792 (2017), ID 080007 [DOI:10.1063/1.4969028]

[19] Abramowski et al., Acceleration of petaelectronvolt protons in the Galactic Centre, Nature, 532 (2016), [DOI:10.1038/nature17147]

[20] Bonnoli, G., et al., for the ASTRI Collaboration and the CTA Consortium, Science with the ASTRI mini-array for the Cherenkov Telescope Array: blazars and fundamental physics, Journal of Physics: Conference Series, 718 (2916), [ID 052004]

[21] Franceschini, A., et al., Very high-energy constraints on the Infrared Extragalactic Background Light, Astronomy \& Astrophysics, 629. (2019), A2, [DOI:10.1051/0004-6361/201935617] 